\title{
Numerical study of a turbulent impinging jet for different jet-to-plate distances using two-equation turbulence models
}

\author{
Julia Wienand $^{1 *}$, Andris Riedelsheimer ${ }^{1}$, Bernhard Weigand ${ }^{1}$
}

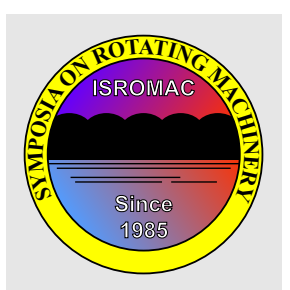

ISROMAC 2016

International

Symposium on Transport

Phenomena and

Dynamics of

Rotating Machinery

Hawaii, Honolulu

April 10-15, 2016

\begin{abstract}
Numerical results of a turbulent impinging jet on a flat plate are compared with experimental reference data from ERCOFTAC. Four different jet-to-plate distances from $H / D=2$ to 14 have been considered at a jet Reynolds number of 23,000. Local flow and heat transfer characteristics are analyzed using the SST turbulence model by Menter and an additional turbulence production limiter by Kato and Launder. For the smaller jet-to-plate distances of $H / D=2$ and 6, the Kato-Launder turbulence production limiter reproduces the heat transfer in the stagnation region very well. At the higher values $H / D=10$ and 14 , the modification underpredicts the reference data.

The near-wall grid resolution at the target plate has been varied for a jet-to-plate distance of $H / D=6$. The results show the influence of the dimensionless distance of the first node near the wall on flow and heat transfer characteristics. A comparison of a hybrid and a block-structured grid assesses the influence on the mesh topology.
\end{abstract}

\section{Keywords}

Impinging jet - RANS turbulence model - Kato-Launder - Heat transfer — Near-wall treatment

${ }^{1}$ Institute of Aerospace Thermodynamics, University of Stuttgart, Germany
${ }^{*}$ Corresponding author: julia.wienand@itlr.uni-stuttgart.de

\section{INTRODUCTION}

Impinging jets are a common cooling technique also widely used in modern aero engines, e.g. as internal cooling systems for turbine vanes and blades or as cooling systems for combustion chamber walls and turbine casings. Achieving high heat transfer coefficients, impingement cooling is an efficient way to counteract locally occurring high heat loads. For cooling large areas, impinging jets are arranged in rows or arrays. This cooling concept finds application as a temperature control for turbine casings. Depending on engine operating conditions, considerable variations of thermal loads lead to a change in blade tip clearances. A reduction of these clearances is essential to increase the efficiency of aero engines. By directing a controlled flow of impinging air onto the turbine casing, clearances remain nearly constant at their optimum. The so-called active-clearance-control (ACC) system consists of several tubes surrounding the turbine. After entering the tubes, coolant air exits via numerous holes directed to the external side of the casing, where it cools the outer casing.

With respect to the highly complex flow mechanisms of impinging jets [1-3], it is necessary to get a deeper understanding of the ACC system. Therefore, we are investigating flow and heat transfer characteristics numerically at the Institute of Aerospace Thermodynamics (ITLR) at the University of Stuttgart. Due to the geometry's complexity and an enormous number of impinging jets, the use of RANS simulations is indispensable. Hence, turbulent structures inside the jets need to be modeled. To make sure that the numerical setup is able to reproduce flow and heat transfer characteristics correctly, a validation is of importance. The European Research Community on Flow, Turbulence and Combustion (ERCOFTAC) [4] provides a detailed set of validated experimental data from literature for a single impinging jet at different jet Reynolds numbers and jet-to-plate distances (Case 25). The heat transfer characteristics for this reference data set have been measured by Baughn and Shimizu [5] and Baughn et al. [6,7]. The detailed description of the flow characteristics is given in Cooper et al. [8].

Several studies discuss the influence of turbulence models with the described reference data set, e.g. [9-11]. Coussirat et al. [9] compared one-, two- and four-equation models varying the jet-to-plate distance $H / D=\{2 ; 6 ; 10\}$ as well as the jet Reynolds number. The v2f model showed the best agreement to the reference data. Beside the $\mathrm{v} 2 \mathrm{f}$ model, the SST turbulence model by Menter [12] was also recommended by Zuckerman and Lior [13] as the best compromise regarding computational speed and accuracy for impinging jet configurations. Draksler and Končar [14] combined the standard SST turbulence model with a turbulence production limiter given by Kato and Launder [15]. They focused their study on a jet-to-plate distance of two. The Kato-Launder limiter improved the numerical accuracy of heat transfer predictions. Vittori [10] compared the KatoLaunder limiter to another limiter called Clip Factor to reduce turbulence kinetic energy, focusing on flow phenomena. 
The work of this paper applies the Kato-Launder production limiter to the available reference data [5-8] for different jet-to-plate distances of $H / D=\{2 ; 6 ; 10 ; 14\}$. The results are compared to the standard SST turbulence model to assess the effect of the Kato-Launder modification. For the lower jet-to-plate distances of $H / D=2$ and 6, flow and heat transfer characteristics are discussed. As for the higher jet-to-plate distances $H / D=10$ and 14 , experimental reference data of the flow-field are not available [8], the focus is set on heat transfer characteristics. The jet Reynolds number was set to 23,000 based on jet diameter and bulk velocity. The second part of this study concerns the influence of the numerical grid on the results at a jet-to-plate distance of $H / D=6$. First, the effect of the dimensionless distance of the first node near the wall $y_{1}^{+}$on flow and heat transfer characteristics is analyzed. Therefore, the near-wall grid resolution at the target plate has been varied to achieve a maximum $y_{1}^{+}$of approx. $0.4-4.3$. A comparison of the results from a hybrid and a block-structured grid indicates the influence on mesh topology. The findings can afterwards be related to the ACC system, where the use of hybrid grids is required due to the geometry's complexity.

\section{NUMERICAL METHOD}

The following section describes the computational domain and the numerical scheme. Hybrid grids were generated with CENTAUR 10.0.2 [16] and block-structured grids with ANSYS ICEM 15.0. For solving the RANS equations ANSYS CFX 15.0 was used. The computations were steady state.

\subsection{Domain and boundary conditions}

The three-dimensional domain contained a single adiabatic tube with an inner diameter of $D=26 \mathrm{~mm}$ and a length of $6 D$. At the tube inlet, a hydrodynamically fully developed flow was applied. The inlet conditions were obtained from a separate computation of a $80 \mathrm{D}$ long adiabatic pipe with a turbulence intensity of $4.1 \%$, a temperature of $T_{j e t}=287.15 \mathrm{~K}$ and a Reynolds number of 23,000. The distance of the tube exit to the target plate varied between $H / D=\{2 ; 6 ; 10 ; 14\}$. As there is no information about the heat flux intensity in the experiments [5-7], in our investigations the temperature of the target plate was set constant at $T_{w}=303.15 \mathrm{~K}$. In the experiments, the temperature of the target plate was in a range of $10 \mathrm{~K}$ [5]. The influence of the exact value was reported to be negligible [5], which was confirmed by Merci et al. [17]. The domain exits of this study were located in a radial distance of $10 D$ and an axial distance of $H+2 D$ to prevent effects on the region of interest $[9,10]$. Opening boundary conditions provided the air to re-enter the domain at a total temperature of $T_{\infty}=287.15 \mathrm{~K}$ and $p_{\infty}=1$ bar. All walls were considered as no-slip boundaries. Figure 1 shows one half of a cut through the computational domain of the single impinging jet. At the walls, 20 prism layers were mounted achieving a dimensionless distance of the first node near the wall of $y_{1}^{+} \leq 1$, which is defined as:

$$
y_{1}^{+}=\frac{u_{\tau} y_{1}}{v}
$$

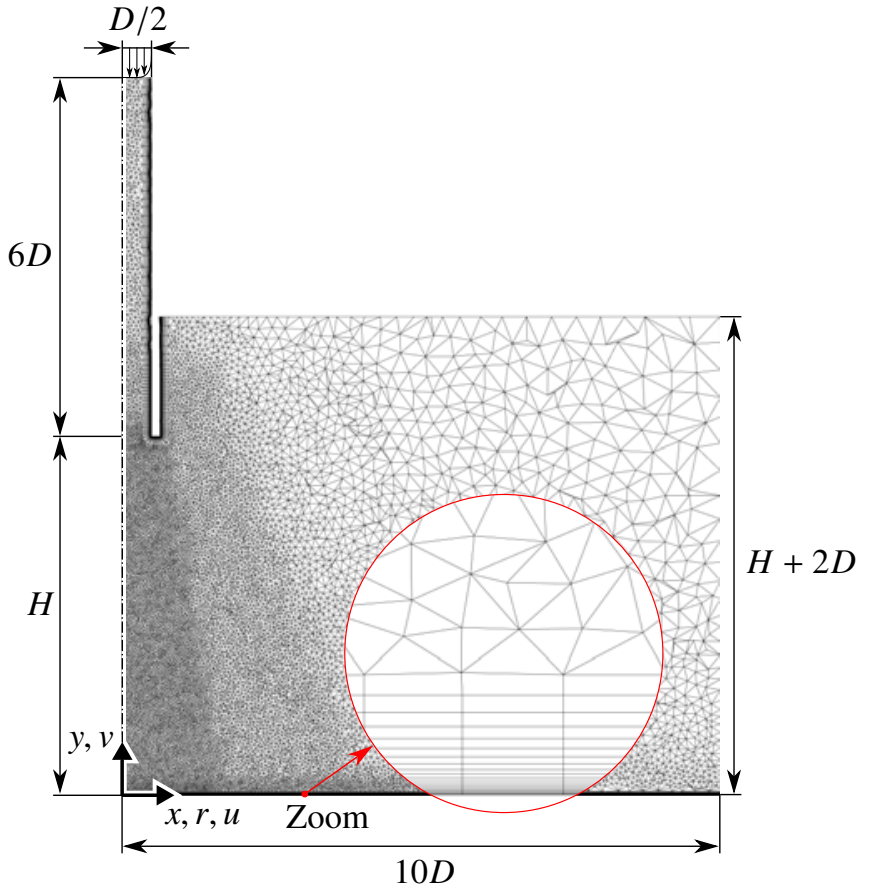

Figure 1. Schematic on the computational domain.

with the shear velocity $u_{\tau}$, the distance of the first node from the wall $y_{1}$ and the kinematic viscosity of the fluid $v$. The rest of the domain was discretized with tetrahedra. Investigating the influence of $y_{1}^{+}$, the height of the first prism layer at the target plate was modified. With a constant total height of the prism layers, the number of layers and the height ratio between the layers was adapted. Table 1 gives an overview of the analyzed configurations. Case B represents the baseline grid described above.

Table 1. Characteristics of the prism layers at the target plate studying the influence of $y_{1}^{+}$, Case B: Baseline grid.

\begin{tabular}{lcccc}
\hline & $\mathrm{A}$ & $\mathrm{B}$ & $\mathrm{C}$ & $\mathrm{D}$ \\
\hline Number of layers & 24 & 20 & 15 & 15 \\
Height ratio & 1.20 & 1.20 & 1.18 & 1.10 \\
$y_{1, \max }^{+}$ & 0.37 & 0.74 & 2.17 & 4.26 \\
\hline
\end{tabular}

\subsection{Thermal and physical properties}

Air was used as coolant and treated as an ideal gas. The molar mass was set to $M=28.96 \mathrm{~g} / \mathrm{mol}$ and the heat capacity at constant pressure to $c_{p}=1004.4 \mathrm{~J} /(\mathrm{kg} \mathrm{K})$, which is valid for dry air at the investigated conditions (VDI 4670). Sutherland's law [18] was used to model dynamic viscosity and thermal conductivity using the coefficients presented by White [19].

\subsection{Governing equations}

Using the RANS equations, turbulence needs to be modeled. Therefore, the standard SST turbulence model by Menter [12] was applied. For incompressible flows like in this study, the 
standard Boussinesq-approximation for the Reynolds-stress tensor characterizes the production of turbulence kinetic energy. In relation to the turbulence viscosity $\mu_{t}$ and the magnitude of the strain rate $S$, the production term $P_{k}$ is expressed as:

$$
\begin{aligned}
& P_{k}=\mu_{t} S^{2} \\
& S=\sqrt{2 S_{i j} S_{i j}}, \quad S_{i j}=\frac{1}{2}\left(\frac{\partial U_{i}}{\partial x_{j}}+\frac{\partial U_{j}}{\partial x_{i}}\right)
\end{aligned}
$$

Reaching high values of $S$ in a stagnation flow, the standard SST turbulence model tends to overpredict turbulent production and thus heat transfer characteristics in the stagnation region of an impinging jet. Kato and Launder [15] suggested a replacement of $S$ using the vorticity rate $\Omega$ :

$$
\begin{aligned}
& P_{k}=\mu_{t} S \Omega \\
& \Omega=\sqrt{2 \Omega_{i j} \Omega_{i j}}, \quad \Omega_{i j}=\frac{1}{2}\left(\frac{\partial U_{i}}{\partial x_{j}}-\frac{\partial U_{j}}{\partial x_{i}}\right)
\end{aligned}
$$

As the deformation in the stagnation region is nearly irrotational, the value of $\Omega$ is close to zero [15]. This leads to a reduction of turbulence production compared to Eq. (2). In a simple shear flow, the replacement of $S$ with $\Omega$ has no effect. Equations (2-5) are implemented by default in the used solver.

\subsection{Numerical accuracy}

Iterative convergence For all computations, the root-meansquare (rms) residuals for the velocity components were in a range of $10^{-4}$ to $10^{-5}$. The other equations achieved an order of magnitude lower. A maximum range of $10^{-4}$ is recommended by Celik et al. [20]. To additionally ensure that iterative convergence is achieved, the absolute values of pressure, velocity and temperature have been monitored at several points in the domain. After showing only slight fluctuations, the computation was assumed to be converged.

Grid convergence The influence of the numerical grid on the results was estimated determing the Grid Convergence Index (GCI). This recommended method was developed by Roache [21]. It is based on the Richardson extrapolation. Celik et al. [20] summarized the procedure to obtain the GCI. We exemplarily evaluated the GCI for a jet-to-plate distance of $H / D=6$ using the standard SST model. For the other cases, the grid was analogous. The numerical grid was systematically coarsened in two steps achieving a refinement factor of $r>1.3$, which is recommended by Celik et al. [20]. The height of the first prism layer was kept constant to avoid an influence of $y_{1}^{+}$. Keeping the total height of the prism layers constant, the number of layers was reduced to 18 for the intermediate and 16 for the coarse grid. The cell growth ratio of the layers was slightly increased. Nusselt numbers showed the highest discretization errors in the stagnation zone with a maximum GCI of $1.3 \%$. The wall-normal velocities in the center-line had a maximum deviation of $14.6 \%$ at the node next to the wall, where the absolute velocity is close to zero. For the wall-parallel velocities, the GCI was below $9 \%$ over all evaluated positions. Normalized rms turbulent fluctuations had the highest discretization error in the center-line with $7.4 \%$. The local discretization error bars are shown in the results section.

\subsection{Reference measurements and experimental uncertainties}

Reference data concerning the flow-field characteristics are given in Cooper et al. [8]. Mean velocities and wall-parallel rms fluctuations $\sqrt{\overline{u^{\prime 2}}}$ were measured with a single wire probe. For the wall-normal rms fluctuations $\sqrt{v^{\prime 2}}$, a cross-wire probe was used. The bulk velocity $U_{b}$ along the pipe was inferred from the center-line velocity $U_{c l}$, which was measured with a pitot tube. The connection is given in White [22]:

$$
U_{b}=U_{c l}(0.811+0.038(\log (R e)-4))
$$

Mean velocities relative to the bulk velocity are estimated to be in a range of $\pm 2 \%$. The uncertainty of $\sqrt{u^{\prime 2}}$ is reported to be within $\pm 4 \%$ and $\sqrt{v^{\prime 2}}$ within $\pm 6 \%$.

Heat transfer characteristics of the electrically heated gold coating on a Plexiglas plate were measured using liquid crystals. Baughn and Shimizu [5] indicated a maximum deviation of $\pm 2.4 \%$ in Nusselt number and $\pm 2.3 \%$ in jet Reynolds number. Baughn et al. [6] reported an accuracy in Nusselt number of $\pm 2.3 \%$ in the stagnation point up to $\pm 3 \%$ for the highest $r / D$.

\section{RESULTS AND DISCUSSION}

The results of the standard SST model are compared to the Kato-Launder modification for all jet-to-plate distances $H / D=\{2 ; 6 ; 10 ; 14\}$, where reference data are available [5-8]. For the smaller jet-to-plate distances, flow and heat transfer mechanisms are discussed. At higher jet-to-plate distances, the focus is set on heat transfer characteristics. At $H / D=6$, the influence of $y_{1}^{+}$and those of the mesh topology are described.

\subsection{Modification of the standard SST model using the Kato-Launder limiter}

Considering the wall-normal velocity $v$ in the center-line $(r / D=0)$ in Fig. 2, there is no influence of the Kato-Launder production limiter neither for $H / D=2$ nor for $H / D=6$.

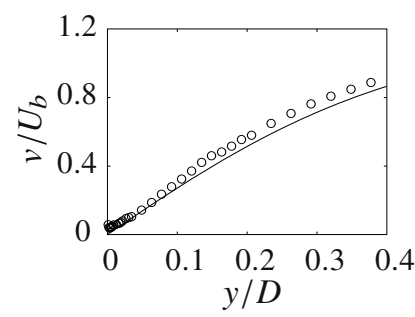

(a) $H / D=2$

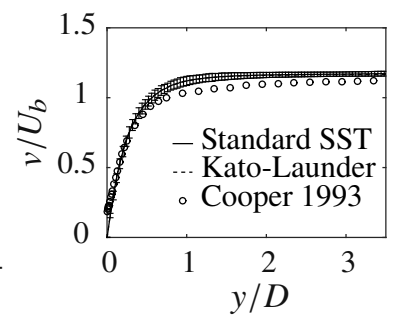

(b) $H / D=6$ including GCI error bars for the standard SST model
Figure 2. Axial mean velocity over the wall distance in the center-line $r / D=0$. 
With a slight underprediction for $H / D=2$ and a slight overprediction for $H / D=6$, the numerical setup represents the experimental data with good accuracy, with and without modification. The bulk velocity was calculated from Eq. (6) analogous to the experiments.

The wall-parallel velocity $u$ in Fig. 3 reveals the increasing influence of the Kato-Launder limiter at higher $r / D$. While

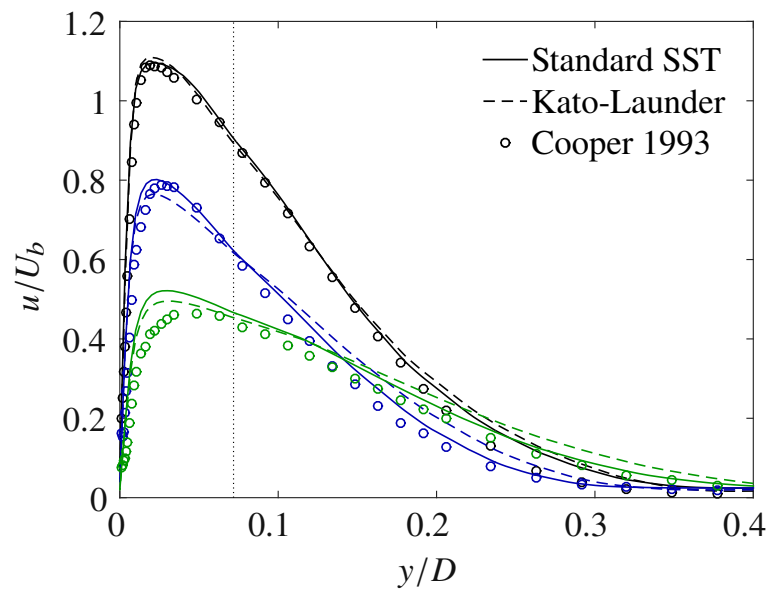

(a) $H / D=2$

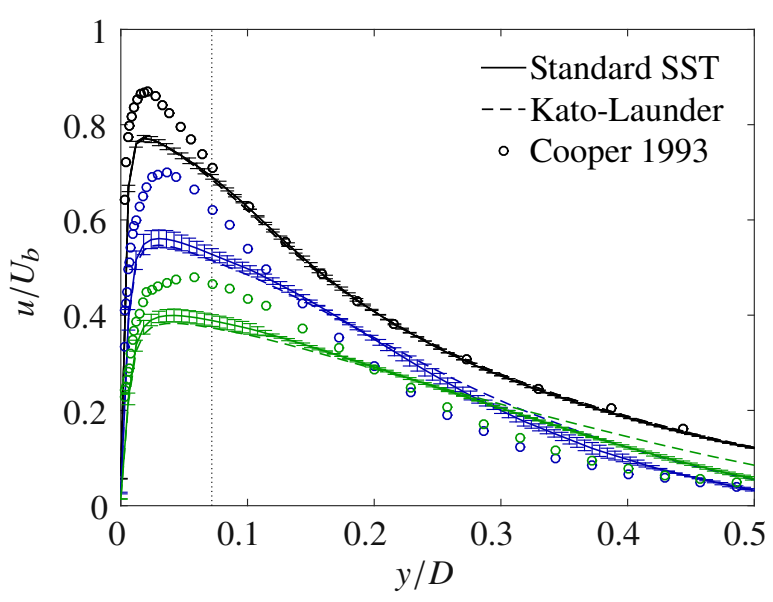

(b) $H / D=6$ including GCI error bars for the standard SST model

Figure 3. Wall-parallel velocity over the wall distance, black: $r / D=1$, blue: $r / D=2$, green: $r / D=3$.

the deviation is almost negligible at $r / D=1$, the curves slightly differ at higher distances. Using the Kato-Launder modification, the velocity near the wall decreases compared to the standard SST model. Further away from the wall it tends to slightly higher values. For $H / D=2$, the experimental results are predicted accurately. For the larger jet-to-plate distance $H / D=6$, the maximum wall-parallel velocity is underpredicted. The overall agreement with the experimental results is slightly better using the standard SST model. The dotted line in Fig. 3 at $y / D \approx 0.07$ indicates the total height of the prism layers of the numerical grid. It is recommended, that the prism layers include the inner part of the boundary layer, which is fulfilled for the present study.

In contrast to the described velocity profiles, there should be a more noticeable influence on the normalized rms turbulent fluctuations, as the Kato-Launder turbulence production limiter modifies the prediction of turbulence kinetic energy. With the use of two-equation turbulence models, turbulent fluctuations are not solved. Nevertheless, to compare the numerical prediction of the turbulent structures with experimental data, they can be calculated from turbulence kinetic energy, assuming isotropic turbulence:

$$
\sqrt{\overline{u^{\prime 2}}}=\sqrt{\overline{v^{\prime 2}}}=\sqrt{\overline{w^{\prime 2}}}=\sqrt{\frac{2}{3} k}
$$

First, we want to analyze the stagnation region of the jet. The normalized rms turbulent fluctuations in the center-line of the jet $(r / D=0)$ and at $r / D=0.5$ are given in Fig. 4 . As it was expected, the Kato-Launder modification shows a huge

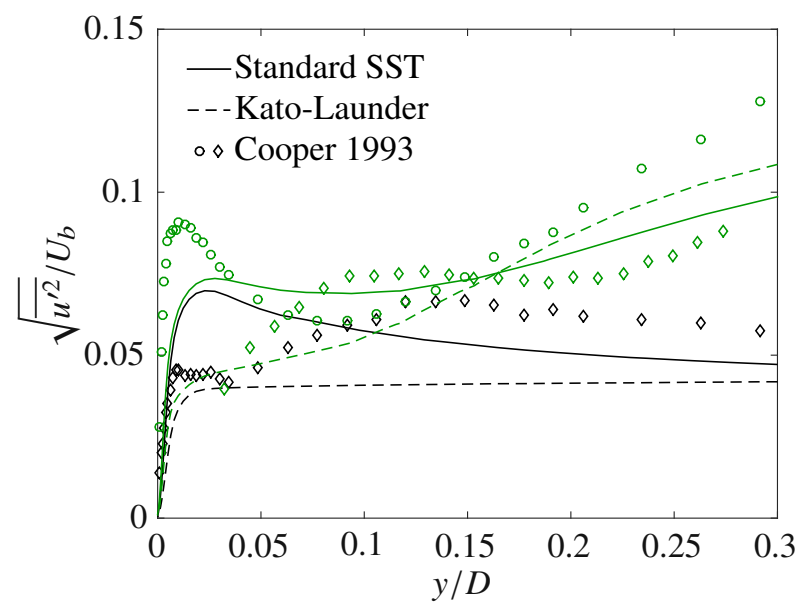

(a) $H / D=2$

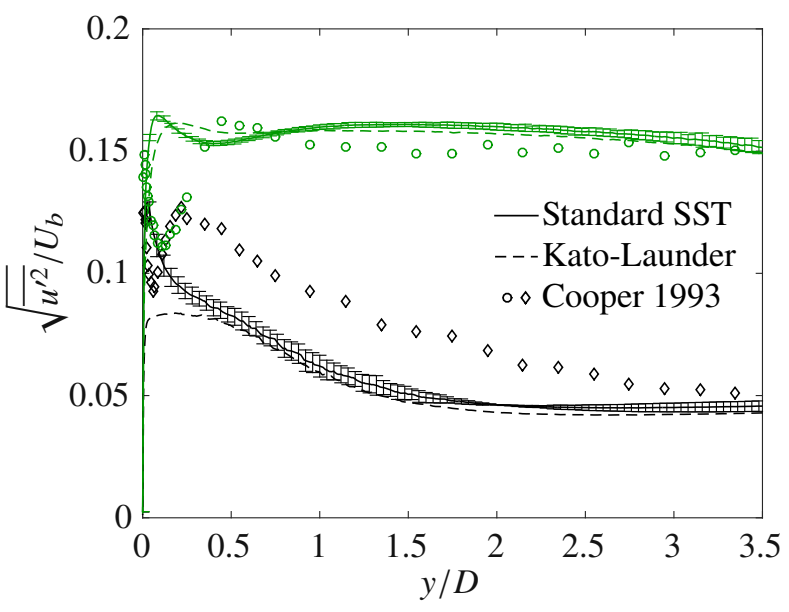

(b) $H / D=6$ including GCI error bars for the standard SST model

Figure 4. Normalized rms turbulent fluctuations over the wall distance in the stagnation region,

black: $r / D=0$, green: $r / D=0.5, \circ \sqrt{u^{\prime 2}}, \diamond \sqrt{\overline{v^{\prime 2}}}$. 
influence on the rms turbulent fluctuations. At $H / D=2$, there is no production of turbulence in the center-line using the limiter. The turbulence intensity has the same value of $4.1 \%$ from the inlet of the tube. Thereby, the amount of turbulence further away from the wall gets underpredicted. In this region, the standard SST model is more accurate compared to the experimental results of wall-normal fluctuations $\sqrt{v^{\prime 2}}$, but overpredicts turbulent structures in the near-wall region. At $r / D=0.5$, Cooper et al. [8] shows experimental results in wall-parallel $\sqrt{u^{\prime 2}}(\circ)$ and wall-normal direction $\sqrt{v^{\prime 2}}(\diamond)$. The difference illustrates the anisopropy of the turbulent structures, which cannot be solved using two-equation models. Near the wall, rms turbulent fluctuations agree better to the higher wall-parallel fluctuations using the standard SST model. With modification, the mean fluctuations are more accurate relating to the wall-normal fluctuations. Wall-normal fluctuations have a big influence on heat transfer characteristics due to the mixing process. An overprediction of these fluctuations can therefore induce an overprediction of heat transfer.

In the center-line at $H / D=6$, the standard SST model predicts the maximum rms turbulent fluctuations of $\sqrt{v^{\prime 2}}$ near the wall very well, whereas the modification underpredicts it. Both numerical results do not show a local decrease of turbulence intensity, as it was seen during the experiments. Further away from the wall, experimental results of $\sqrt{v^{\prime 2}}$ are underpredicted with and without modification. At $r / D=0.5$, the local minimum in the experiments is even larger than in the center-line. Both models overpredict turbulence in this region, showing no local decrease. The standard SST model has a steeper gradient than using the Kato-Launder limiter, but it decreases earlier. Further away from the wall, rms turbulent fluctuations of $\sqrt{\bar{u}^{\prime 2}}$ are predicted well.

Figure 5 shows the normalized rms turbulent fluctuations in the wall-jet region at $r / D=2$ and 3. The use of the turbulence limiter leads to higher rms turbulent fluctuations than the standard SST model. Showing the same value near the wall, the numerical results differ with increasing wall distance. The experimental profile of $\sqrt{u^{\prime 2}}$ in the near wall region is underpredicted with and without modification. But keeping in mind that the fluctuations in wall-normal and also in azimuthal direction are smaller in the developing wall jet [23], the prediction relating to all spatial directions cannot be assessed. For $H / D=2$, the smaller value in wall-normal direction $\sqrt{\sqrt{v^{\prime 2}}}(\diamond)$ compared to the radial direction $\sqrt{\sqrt{u^{\prime 2}}}(\circ)$ can be seen at $r / D=3$. For $r / D=2$ and $H / D=6$ no experimental reference data are given in Cooper et al. [8].

To clarify in which regions the Kato-Launder limiter has an influence on turbulence, the deviation of turbulence kinetic energy $\Delta k$ with (KL) and without modification (SST) is depicted in Fig. 6 for all jet-to-plate distances. The deviation is shown in relation to the maximum deviation $\Delta k_{\max }$ :

$$
\frac{\Delta k}{\Delta k_{\max }}=\frac{k_{S S T}-k_{K L}}{\left\{k_{S S T}-k_{K L}\right\}_{\max }}
$$

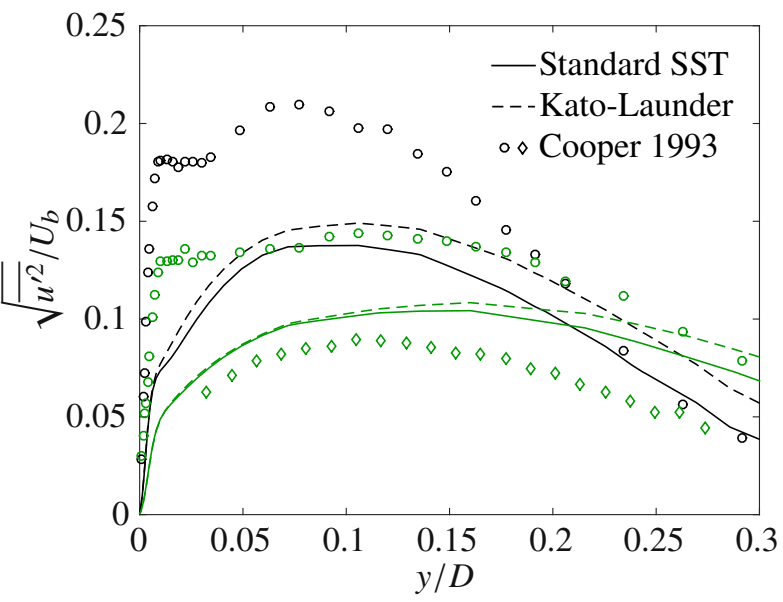

(a) $H / D=2$

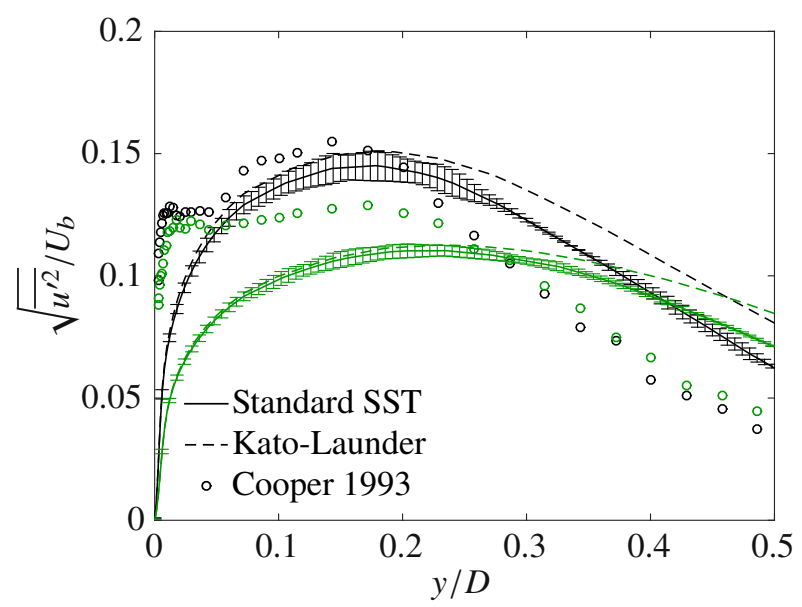

(b) $H / D=6$ including GCI error bars for the standard SST model

Figure 5. Normalized rms turbulent fluctuations over the wall distance in the wall-jet region,

black: $r / D=2$, green: $r / D=3, \circ \sqrt{u^{\prime 2}}, \diamond \sqrt{v^{\prime 2}}$.

The maximum deviation is always based on the domain of each configuration. This has to be kept in mind when comparing the results of the configurations to each other. Positive values (black) of the quantity given by Eq. (8) show the regions, where the Kato-Launder limiter reduces $k$, while it increases $k$ in the regions with negative values (red). A reduction is achieved in the stagnation region of each configuration. In the free jet region, there is a negligible deviation for $H / D=6$. We assume, that the deviation is due to a slightly different convergence of the simulations with and without modification. For the higher jet-to-plate distances, there is no effect of the modification in the shear layer of the free jet, as it was depicted in [15]. In the wall-jet region, $k$ is predicted higher with modification. For $H / D=2$, the amount of increase in $k$ compared to the standard SST model is in the same range as the decrease in the stagnation region. In this case, $k$ increases also in close proximity to the wall at $1.5<x / D<2$. While this effect is less for $H / D=6$, an increase in $k$ due to the 


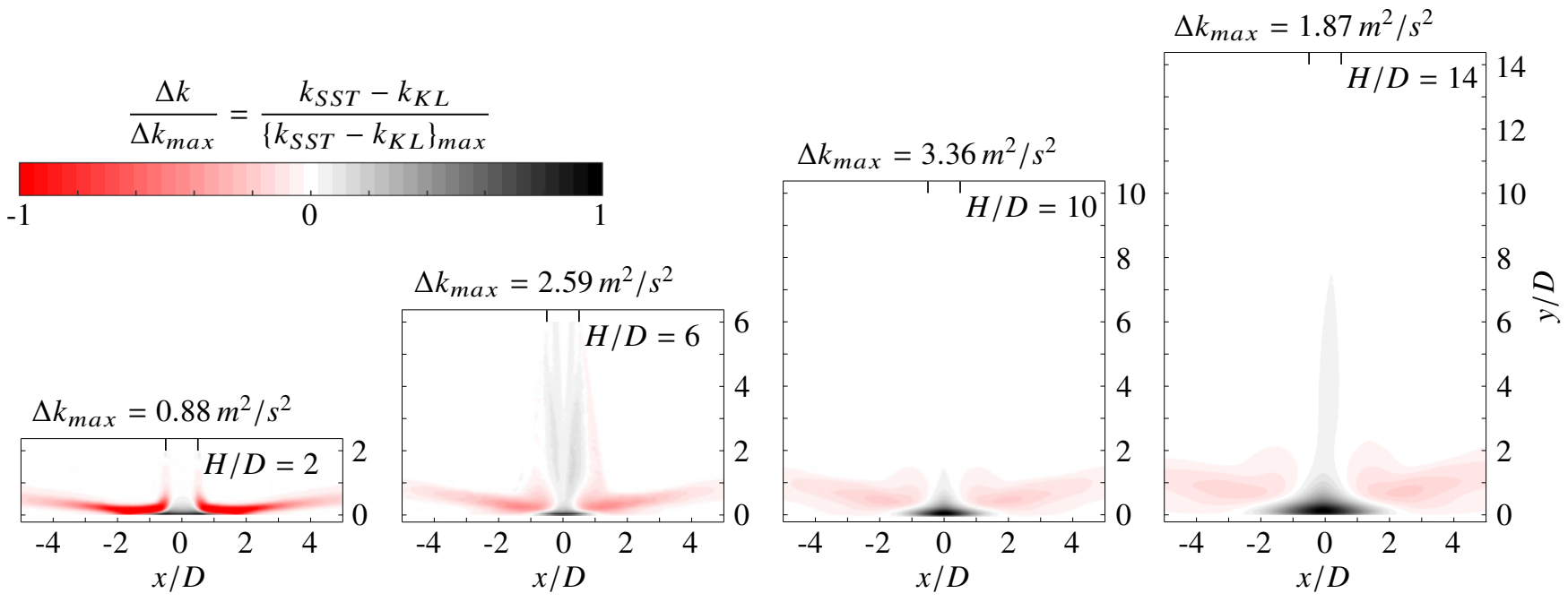

Figure 6. Deviation of turbulence kinetic energy with (KL) and without (SST) Kato-Launder production limiter.

limiter occurs only further away from the wall for the higher jet-to-plate distances. The increase in $k$ is effected by the higher vorticity rate $\Omega$ (Eq. (5)) compared to the strain rate $S$ in the regions with an increasing amount of turbulence.

After describing the flow-field, we want to analyze heat transfer characteristics. Therefore, the Nusselt number $N u$ is evaluated, which is defined as:

$$
N u=\frac{\dot{q}_{w} D}{k\left(T_{w}-T_{j e t}\right)}
$$

with the heat flux density at the wall $\dot{q}_{w}$, thermal conductivity $k$, temperature at the target plate $T_{w}$ and jet temperature $T_{j e t}$. The use of $T_{j e t}$ as a reference temperature of the fluid is valid, as it correlates to the outer air temperature and the jet Reynolds number is below 35,000 [3].

Nusselt numbers over the radial distance for the investigated jet-to-plate distances are given in Fig. 7. In the stagnation region, the use of the Kato-Launder limiter leads to a reduction

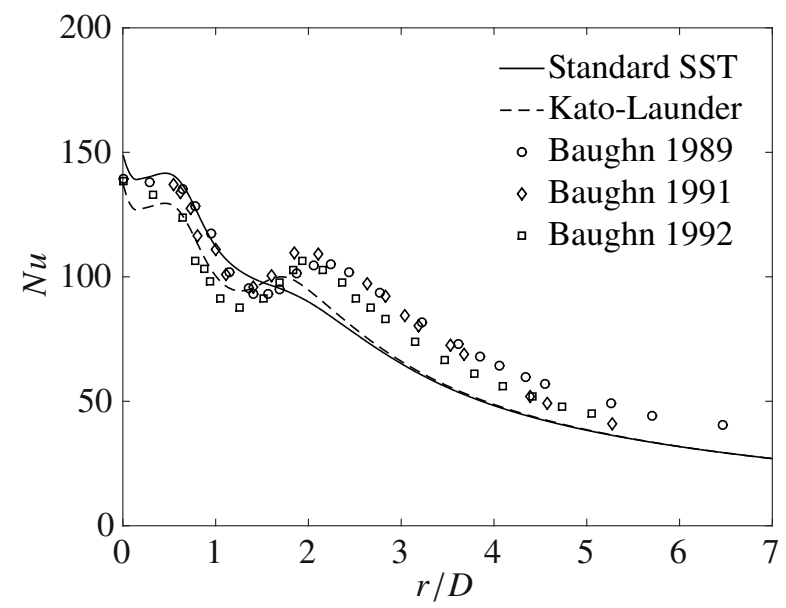

(a) $H / D=2$ of $N u$ compared to the standard SST model for all jet-to-plate distances. This can be explained with the lower production of $k$ using the modification. For $H / D=2$, the heat transfer is slightly overestimated by the standard SST model, whereas the modification slightly underestimates it. For both models, $\mathrm{Nu}$ decreases with a similar gradient at $0.7<r / D<1.3$, where the results are in good agreement to the reference data. While $\mathrm{Nu}$ continues to decrease with the standard SST model, the Kato-Launder limiter shows a secondary peak in the Nusselt number distribution, as it was already reported by Draksler and Končar [14]. A secondary peak was also seen in the reference experiments [5-7]. The appearance of this secondary peak is related to anisotropic turbulent effects in the developing wall jet. Uddin et al. [23] explained the increasing heat transfer with an increasing velocity due to a break up of a ring vortex. As anisotropic effects are not incorporated in the two-equation models, the Kato-Launder modification does not solve the physical phenomena, which induces this secondary peak. The numerical prediction of the peak is related to an increase in

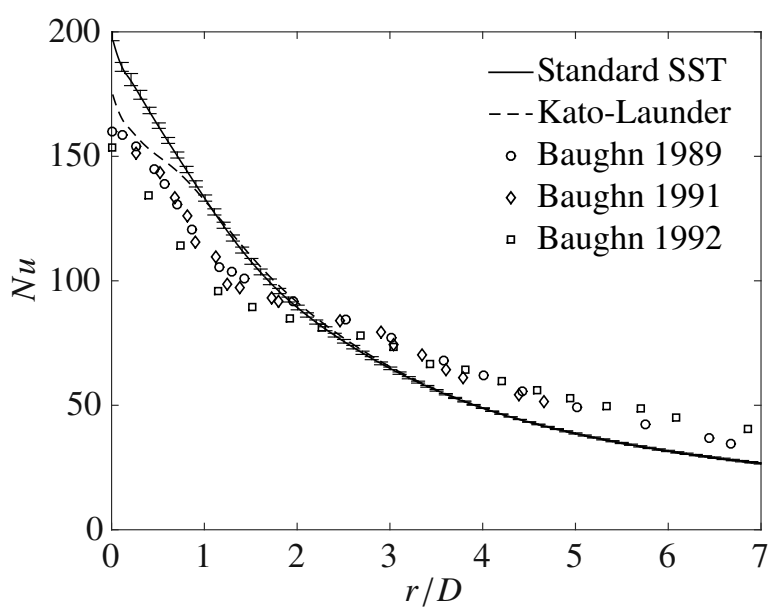

(b) $H / D=6$ including GCI error bars for the standard SST model 


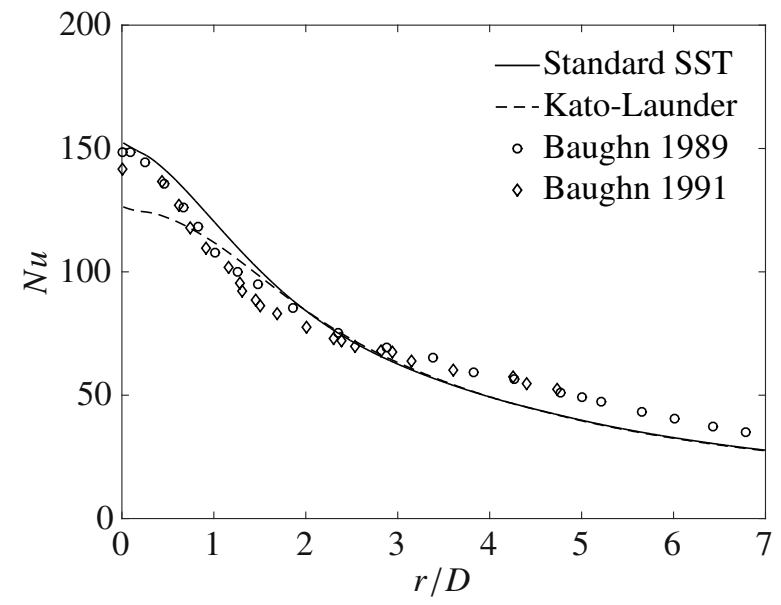

(c) $H / D=10$

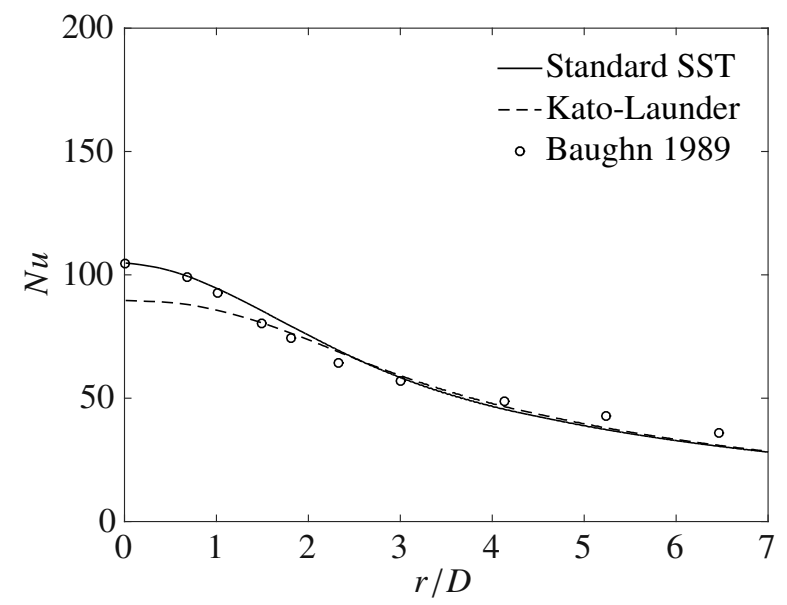

(d) $H / D=14$

Figure 7. Nusselt number distributions along the target plate.

$k$ (Fig. 6). In comparison to the standard SST model, the Kato-Launder modification calculates the production of $k$ (Eq. (4)) with respect to the vorticity rate $\Omega$. An increase of $\Omega$ in the region of the vortex break up leads to the increase in $k$ and therefore to the secondary peak. Although the physical phenomena cannot be resolved completely, we assume the Kato-Launder modification to indicate the turbulent effects causing this secondary peak in the reference experiments.

At $H / D=6$, the standard SST model overestimates heat transfer in the stagnation region. Using the Kato-Launder limiter, this overestimation is reduced, but the profile of $N u$ is less accurate with reference experiments. One explanation of the deviation to the experimental results is an overprediction of the potential core length. According to Han and Goldstein [3], the potential core length of an impinging jet is $4 D-6 D$. Georgiadis et al. [24] reported the standard SST model to overpredict the length of the potential core. Assuming that in the experiments, the potential core ended downstream the target plate, it still reaches the plate using the SST model with and without modification. We believe the higher potential core length to induce the overestimation of heat transfer in the stagnation region.

For the higher jet-to-plate distances of $H / D=10$ and 14 , the standard SST model reproduces experimental data accurately. The usage of the Kato-Launder limiter induces an underprediction of heat transfer in the stagnation region. Despite the overprediction of the potential core length, the center-line velocity of a free jet is predicted accurately at a distance of $10 D-16 D$ from the nozzle exit due to an overprediction of the mixing rate [24]. For $H / D=10$ and 14 , the velocity of the impinging jet is assumed to be similar to the reference experiments. Therefore, the prediction of heat transfer in the stagnation region is in good accordance to the reference data using the standard SST model. The reduction of $k$ using the modification leads to a reduction of $N u$ and therefore to an underprediction of heat transfer in the stagnation region.

\subsection{Near-wall grid resolution}

In the next section, we want to discuss the influence of the near-wall grid resolution on flow and heat transfer predictions. Four numerical grids were investigated, varying the height of the first prism layer, as it was described in Tab. 1. In Fig. 8, the dimensionless distance of the first node near the wall $y_{1}^{+}$is shown over the radial distance.

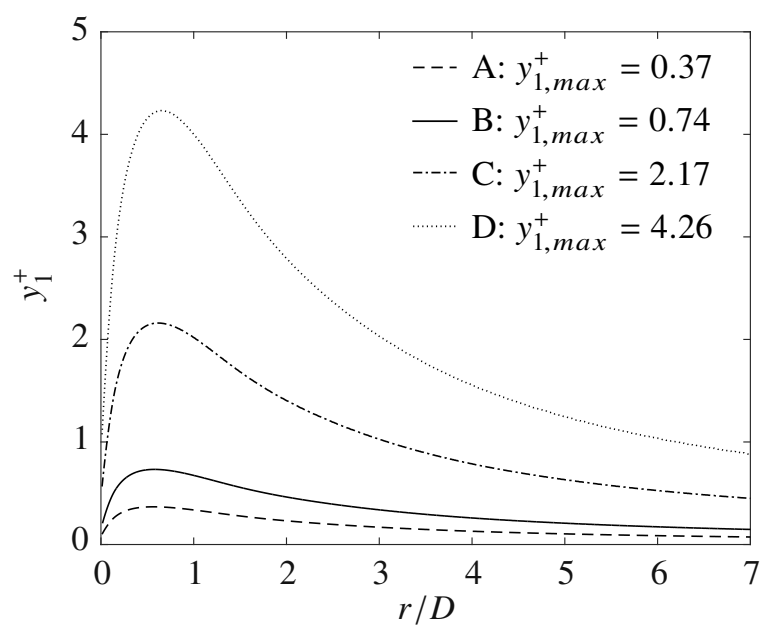

Figure 8. Dimensionless distance of the first node near the wall along the target plate varying $y_{1}^{+}$, A-D see Tab. 1 .

Mean velocities and normalized rms turbulent fluctuations are not influenced by the near wall grid resolution in the investigated range of $y_{1}^{+}$. They are similar for all configurations.

An influence of $y_{1}^{+}$was observed in relation to heat transfer predictions. The distributions of $\mathrm{Nu}$ in the stagnation region are given in Fig. 9. The results become similar at higher radial distances, which are therefore not shown. While the influence is negligible for $y_{1}^{+}<1$ comparing the results of case $\mathrm{A}$ and $\mathrm{B}$, deviations occur at higher values of $y_{1}^{+}$with case $\mathrm{C}$ and $\mathrm{D}$. Case $\mathrm{C}$ predicts a reduced heat transfer in the stagnation region. 


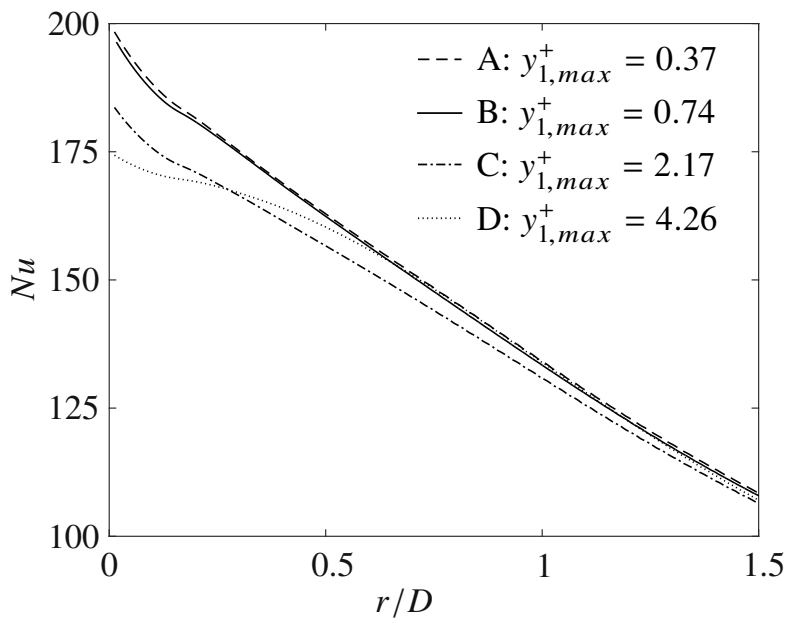

Figure 9. Nusselt number distributions along the surfaces varying $y_{1}^{+}$, A-D see Tab. 1 .

Analyzing Case $\mathrm{D}$, the value of $N u$ is further reduced and the profile of $N u$ is predicted differently. Even though the cases with poor grid-resolution near the wall correspond better to the experiments, they are not recommended. Grid-resolution should not influence the results. Therefore, the results of this study should not be compared to experimental data.

\subsection{Mesh topology}

Investigating the influence of mesh topology on the numerical results, an additional block-structured grid was generated using ANSYS ICEM 15.0 for a jet-to-plate distance of $H / D=6$. The height of the first cells at the wall and the height ratio in the near wall region corresponded to those of the hybrid grid.

Predictions concerning velocity profiles are similar with both mesh topologies. Only in the free jet region slightly higher values occur using the block-structured grid. According to the solver manual, unstructured grids tend to have greater numerical diffusion compared to structured ones, which can explain the differences in jet velocity.

Heat transfer predictions show larger deviations. Heat transfer in the stagnation region is lower with the blockstructured grid. This can be seen in Fig. 10. With increasing radial distance, the results become similar. The origin of the discrepancies is the different prediction of $k$. Higher values of up to $45 \%$ in the center-line occur using the hybrid grid. Although the differences are considerable compared to the jet velocity, we assume, that these discrepancies are also present due to larger numerical diffusion using the hybrid grid.

\section{CONCLUSIONS}

We investigated a turbulent impinging jet for different jet-toplate distances $H / D=\{2 ; 6 ; 10 ; 14\}$ combining the standard SST turbulence model by Menter [12] with a turbulence production limiter given by Kato and Launder [15]. Numerical results were compared to experimental reference data [5-8] from the ERCOFTAC database [4]. Flow-field characteristics

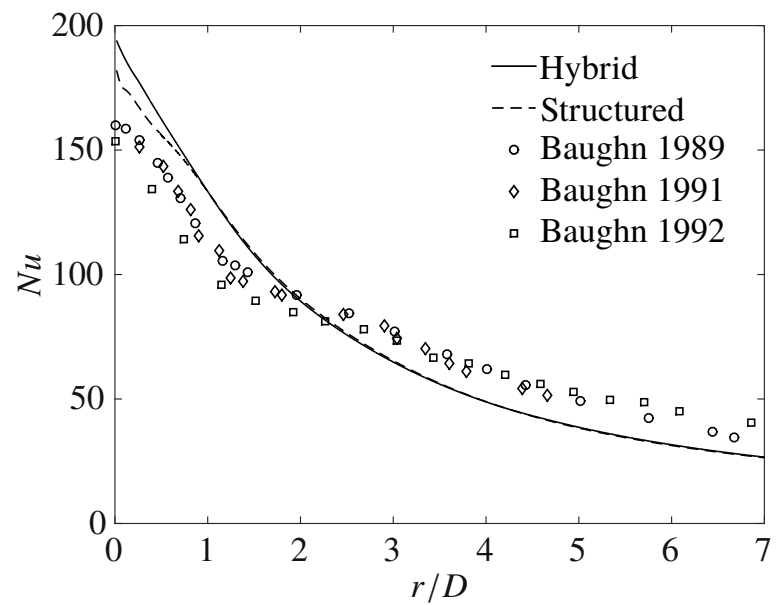

Figure 10. Nusselt number distributions along the surfaces from a hybrid and a block-structured grid.

were described only for the lower jet-to-plate distances $H / D=$ 2 and 6 , whereas heat transfer phenomena were discussed for all configurations. The influence of the limiter on velocity prediction is low. High accuracy to experimental data is achieved for wall-normal and wall-parallel velocities. Only the maximum wall-parallel velocity in the near-wall region is underpredicted with $H / D=6$. The numerical prediction of normalized rms turbulent fluctuations changes using the Kato-Launder modification. The fluctuations decrease in the stagnation region of the jet compared to the standard SST model, but slightly increase in the wall-jet region. Hence, the overprediction of turbulence intensity of the impinging jet is reduced by the limiter. Reducing turbulent structures, the Kato-Launder modification also reduces the prediction of heat transfer in the stagnation region. For the lower jet-toplate distances of $H / D=2$ and 6 , experimental results are reproduced more accurately. Using the limiter, a secondary peak of the Nusselt number is predicted for $H / D=2$ due to a local increase in turbulence kinetic energy. At the higher jet-toplate distances of $H / D=10$ and 14, heat transfer predictions using the standard SST model are accurate to the experimental results, whereas the Kato-Launder modification leads to an underestimation in the stagnation region. It thus depends on the jet-to-plate distance, if the use of the Kato-Launder production limiter improves the prediction of heat transfer for an impinging jet. For higher values, like they occur in activeclearance-control (ACC) systems, we do not recommend the Kato-Launder modification.

In the second part of this study, the influence of the dimensionless wall-distance of the first node was discussed. We analyzed four configurations with different near-wall grid resolutions $\left(0.4<y_{1, \max }^{+}<4.3\right)$. In this range, no remarkable influence on the flow-field was observed. Heat transfer predictions in the stagnation region decrease with lower near-wall grid resolution. As the influence is negligible for $y_{1}^{+}<1$, we recommend to be below this value while investigating heat transfer phenomena. 
The comparison of a hybrid and a block-structured grid showed a slight deviation in jet velocity, but higher discrepancies of heat transfer characteristics in the stagnation region. Due to greater numerical diffusion of a hybrid grid, the velocity in the free jet region is slightly lower. In the stagnation region, turbulence kinetic energy is predicted lower with the block-structured grid. Therefore, heat transfer in the stagnation region decreases. The lower values of the Nusselt number accord better to the experimental reference data.

\section{ACKNOWLEDGMENTS}

The financial support for this work by the MTU aero engines $\mathrm{AG}$ and the permission to publish this paper are highly acknowledged.

\section{REFERENCES}

[1] H. Martin. Heat and Mass Transfer between Impinging Gas Jets and Solid Surfaces. Advances in Heat Transfer, Academic Press, New York, 13:1-60, 1977.

[2] R. Goldstein, K. Sobolik, and W. Seol. Effect of Entrainment on the Heat Transfer to a Heated Circular Air Jet Impinging on a Flat Surface. Journal of Heat Transfer, 112:608-611, 1990.

[3] B. Han and R. J. Goldstein. Jet-Impingement Heat Transfer in Gas Turbine Systems. Annals of the New York Academy of Sciences, 934:147-161, 2001.

[4] European Research Community on Flow, Turbulence and Combustion. Case 25: Normally-Impinging Jet from a Circular Nozzle. www . ercoftac.org/products_and_ services/classic_collection_database, 1992.

[5] J. Baughn and S. Shimizu. Heat Transfer Measurements From a Surface with Uniform Heat Flux and an Impinging Jet. Journal of Heat Transfer, 111:1096-1098, 1989.

[6] J. Baughn, A. Hechanova, and Xiaojun Yan. An Experimental Study of Entrainment Effects on the Heat Transfer from a Flat Surface to a Heated Circular Impinging Jet. Journal of Heat Transfer, 113:1023-1025, 1991.

[7] J. Baughn, X. Yan, and M. Masbah. The Effects of Reynolds Number on the Heat Transfer Distribution from a Flat Plate to an Impinging Jet-Impingement. ASME Heat Transfer Division: Fundamental and Applied Heat Transfer Research for Gas Turbine Engines, 226:1-7, 1992.

[8] D. Cooper, D. Jackson, B. Launder, and G. Liao. Impinging jet studies for turbulence model assessment. Part I: Flow-field experiments. International Journal of Heat and Mass Transfer, 36:2675-2684, 1993.

[9] M. Coussirat, J. van Beeck, M. Mestres, E. Egusguiza, J.-M. Buchlin, and X. Escaler. Computational Fluid Dynamics Modeling of Impinging Gas-Jet Systems: I. Assessment of Eddy Viscosity Models. Journal of Fluids Engineering, 127(4):691-703, 2005.
[10] F. Vittori, L. Rojas-Solórzano, and M. Pavageau. Comparative Study of RANS Turbulence Models for Impinging Jet Simulations. Annual Congress of Engineering ASME USB, 1:1-8, 2011.

[11] A. Abdon and B. Sundén. Numerical Investigation of Impingement Heat Transfer using Linear and Nonlinear Two-Equation Turbulence Models. Numerical Heat Transfer, Part A: Applications, 40(6):563-578, 2001.

[12] F. Menter. Two-Equation Eddy-Viscosity Turbulence Models for Engineering Applications. AIAA Journal, 32(8):1598-1605, 1994.

[13] N. Zuckerman and N. Lior. Impingement Heat Transfer: Correlations and Numerical Modeling. Journal of Heat Transfer, 127:544-552, 2005.

[14] M. Draksler and B. Končar. Analysis of heat transfer and flow characteristics in turbulent impinging jet. Nuclear Engineering and Design, 241(4):1248 - 1254, 2011. International Conference on Nuclear Energy for New Europe 2009.

[15] M. Kato and B. E. Launder. The Modelling of Turbulent Flow around Stationary and Vibrating Square Cylinders. Proceedings of the 9th Symposium on Turbulent Shear Flows, 1993.

[16] CENTAUR Software. WWW. centaursoft. com.

[17] B. Merci, J. Vierendeels, C. De Langhe, and E. Dick. Numerical simulation of heat transfer of turbulent impinging jets with two-equation turbulence models. International Journal of Numerical Methods for Heat \& Fluid Flow, 13(1):110-132, 2003.

[18] W. Sutherland. The viscosity of gases and molecular force. Philosophical Magazine Series 5, 36(223):507$531,1893$.

[19] F. M. White. Viscous Fluid Flow. McGraw-Hill Book Company, 2 edition, 1974.

[20] I. B. Celik, U. Ghia, P. J. Roache, C. J. Freitas, H. Coleman, and P. E. Raad. Procedure for Estimation and Reporting of Uncertainty Due to Discretization in CFD Applications. Journal of Fluids Engineering, 130:0708001-1-07080014, 2008.

[21] P. J. Roache. Perspective: A Method for Uniform Reporting of Grid Refinement Studies. Journal of Fluids Engineering, 116:405-413, 1994.

[22] F. M. White. Fluid Mechanics. McGraw-Hill Book Company, 2 edition, 1986.

[23] N. Uddin, S. O. Neumann, and B. Weigand. LES simulations of an impinging jet: On the origin of the second peak in the Nusselt number distribution. International Journal of Heat and Mass Transfer, 57(1):356 - 368, 2013.

[24] N. J. Georgiadis, D. A. Yoder, and W. B. Engblom. Evaluation of Modified Two-Equation Turbulence Models for Jet Flow Predictions. AIAA Journal, 44(12):31073114, 2006. 